\title{
Manage digital assets with ITIL: Improve product configurations and service management
}

\author{
Cameron A. Fisher \\ provides enterprise consulting in the areas of IT service management and IT portfolio management.
}

Keywords: ITIL, contact centers, consumer devices, customer service

\begin{abstract}
Information Technology Infrastructure Library (ITIL) techniques are not widely known for external customer-facing situations. Relatively few marketers are aware of the importance of configuration management to the user experience, service support and overall CRM delivery. New tools and methods available yield significant value from automated inventorying, deployment and management of IT enabled devices.
\end{abstract}

\section{INTRODUCTION}

IP-addressable devices are increasingly common across many types of consumer and business activities. In this paper, the case is made for applying techniques inspired by the Information Technology Infrastructure Library (ITIL) to improve management of the technical and business lifecycle of consumer devices. Organizations should apply these ITIL principles to better manage contact centers and customer service. ITIL should not be limited solely to internalfacing infrastructure matters. Effective digital asset management is central to overall service management, customer satisfaction and business strategy.

Opportunities to attain improvements in operations, support and service level management are being seized by many organizations. Similarly, outsourcing providers are investing in service management and asset management (eg hardware, software and contract) best practices. When organizations realize these new process efficiencies using ITIL, the result is better service and profits.

The impact of ITIL upon enterprise information technology management is gaining proponents. Adoption of ITIL principles helps refine IT best practices. Client systems management and configuration management of devices are improved. Change management can be streamlined. In fact, ITIL-influenced practices need not be constrained solely to infrastructure equipment (such as mainframes, servers, PCs, routers and switches).

ITIL can help drive productivity gains, cost savings and stakeholder wins more broadly than many people realize.

Indeed, infrastructure support is vital to service delivery and overall service management. By adopting the ITILinspired discipline and processes, the business (not just the infrastructure) will perform better and yield important gains by leveraging the "configuration management database". This helps 
synchronize contact centers and customer touch points. Aligning IT initiatives to business needs improves the efficacy of IT spending. In some cases, the boundary of IT infrastructure and what constitutes a strategic business undertaking can merge. Service delivery and service support become tactical weapons that improve overall product strategy and profits. Consider:

- In Florida, soft-drink firms equip vending machines with climate temperature sensors and remote price setting mechanisms. Since consumer's price resistance diminishes at higher outdoor temperatures, higher prices or special discounts can dynamically occur. The status of pricing, stockout alerts, replenishment orders or malfunction self-healing can be transmitted dynamically in real time to support centers. The resulting impact on return on assets is dramatic.

- McDonald's restaurants offer \$1 DVD rentals from in-store vending machines. No doubt the contact center will field problem-fix incidents, as opposed to onsite employees who are busy making fries and handling operations. McDonald's DVD machines, like Harrah's slot machines, contain data and equipment that need to be dynamically monitored and the delivery and support (contact centers) mechanisms can be optimized via empowered contact centers.

- GPS and IP enabled automobiles exemplify the many types of business and consumer activities that will require configuration management. The mobile "automobile-office" with enhanced displays, voice-recognition and handsfree computing functionality will be common for both the operation and maintenance repair aspects. Applyng
ITIL-inspired techniques to manage the technical and business lifecycle of such devices is projected to grow considerably.

\section{TRENDS AND DRIVERS FOR CONFIGURATION MANAGEMENT OF CONSUMER DEVICES}

Today's product and service offerings are increasingly complex. This complexity is increasingly supported by semiconductors, micro-processors and IP-enabled capabilities. The hardware is increasingly software application dependent. Tools and techniques are available to contact centers that will support performance optimization of many of these IP-enabled devices.

Examples of demand for pre- and post-sales services support for IT enabled products include:

- Apple iPod and related peripherals;

- Xbox and PlayStation;

- Oversized, thin screen and highdefinition televisions (HDTVs), and other entertainment appliances (eg TiVo, set-top box, etc);

- Self-service DVD rental kiosks;

- Cellular phones, PDAs and hybrids (eg Treo, Blackberry);

- Auto dealership diagnostic and repair systems;

- Casino gambling consoles or slot machines;

- ATMs, banking and financial service machines (over 16,000 ATMs operated by Bank of America);

- Airline ticket kiosks;

- Home, office and consumer computers.

For example, the Best Buy retailer chain established a "Geek Squad" to assist customers with a range of techno-services 
including dealing with the configuration complexity of consumer electronics, home computers and extended warranties.

Customers often need to contact manufacturers and service providers to access subject matter experts and support resources. This need has spawned armies of contact center employees that operate around-the-clock, and around-the-globe to perform a mission-critical activity that sustains customer satisfaction levels.

In many instances, the GUI is the customer experience as well as the brand impression. The success of the Apple iPod and the Apple Nano illustrate the importance of the device interface and product design. ITIL can help the configuration management and the support of such devices. Service delivery and support are likewise vital to GUI performance and usability/reliability. Hence, a successful contact center will bolster the technical and service aspects of the customer experience, and improve the overall value derived from the total solution.

Internationally accepted standards such as ITIL promote interoperability and standardization of processes, people and technologies. The combination of ITIL, contact centers and teleworkers is a powerful combination for customer support strategies. The growing availability of fiber-optics and multimedia (such as voiceover IP (VOIP), video, email, online chat, etc) is further driving new applications, business rules and efficiencies among contact center operations and the related smart devices that the contact centers serve and support.

\section{CONTACT CENTERS}

ITIL can help customers and organizations raise the quality of service and reduce total costs of usage and support. For inbound customers service desks as well as outbound direct marketing centers, the "center" is often a disparate collection of networked or home-based workers based in different physical locations. (The phrase 'homeshoring' suggests a call center might increasingly be at a North American kitchen table, to gain productive workers with low turnover). The workers may never actually be at a "center," nor be physically near customers, supervisors, partners or suppliers. How will they be coordinated effectively? How will these workers receive suppport from the organizations or provide support to the stakeholders and end-users? ITIL standards can make a positive impact.

To install, migrate, upgrade and troubleshoot IT enabled devices in a fast paced environment without a disciplined approach will quickly overwhelm an organization. In a distributed environment of complex and configuration-intensive devices it makes sense to adopt many of the ITIL-inspired best practices in support of users, and thereby realize service and cost improvements.

\section{CONTACT CENTERS AND IT SERVICE MANAGEMENT}

Often referred to as "call centers," contact centers' communication infrastructure now relies less on telephones. The abundance of transnational and transoceanic fiberoptics as well as growing capabilities in VOIP has contributed to potent, and affordable bandwidth. Contacts can occur asynchronously via internet, cable operator, webcam, tele-video, voicemail, ATMs or a range of IP addressable digital technologies. Increasingly, vendors offer solutions which incorporate caller ID, RFID, speech recognition, screen-pop scriptings, 64-bit 


\section{External Facing, End User Customers *}

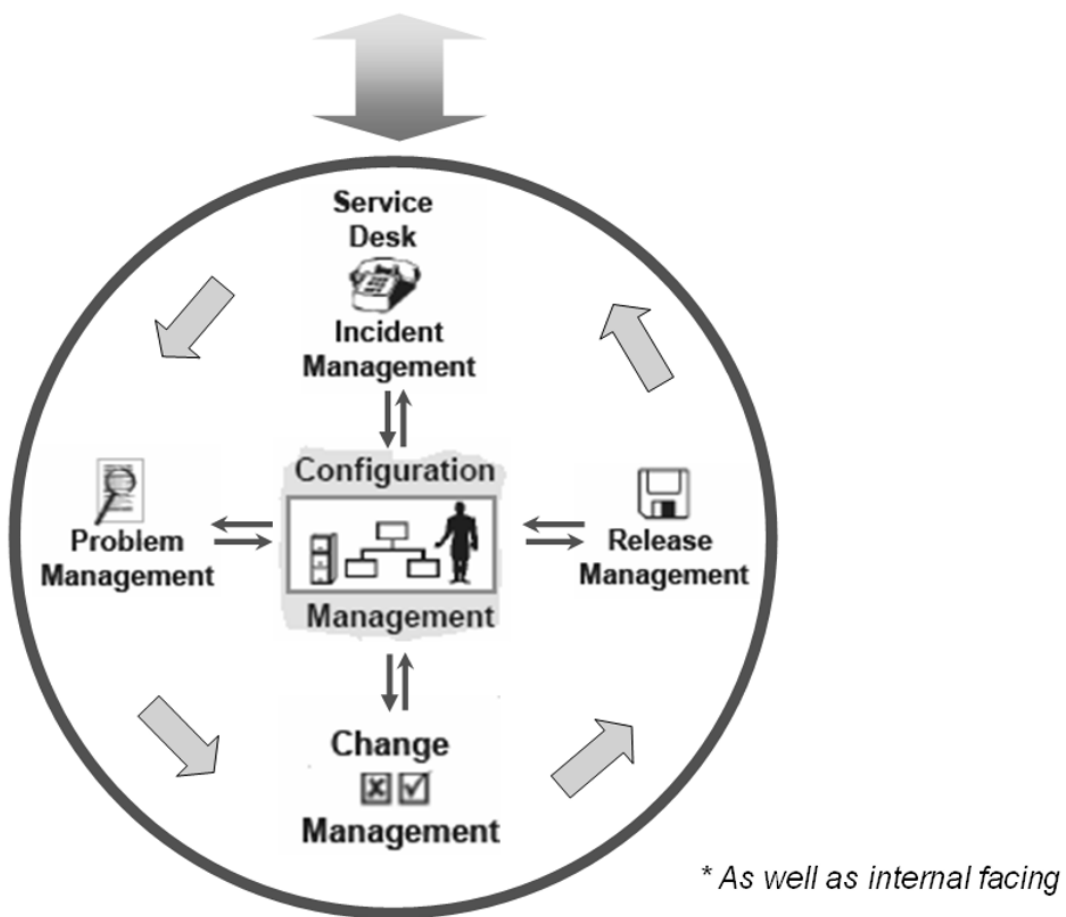

Figure 1: External facing, end user customers

computing and a variety of technologies that yield new capabilities, efficiencies and challenges.

As partners, suppliers and customers increasingly use broadband-enabled IP addressable equipment, contact centers using ITIL can deliver multichannel and multimedia services more effectively. Consider a sample scenario where a contact center supervisor uses a single console to multitask with a customer or partner to:

- View email history.

- Monitor agent workload levels. Route an escalation to divert bottlenecks and minimize mean-time-to-resolution.

- View buying history and tap into CRM systems or online product catalogs.

- Collaborate a best mix of customerfacing agent representatives with expert support teams. Match lucrative customers to most capable/available agents and route request/incident accordingly. Leverage business rules (eg select most appropriately skilled or most availabe agent) to reduce costs and mean time to resolution.

- Adapt quickly to changes in organization, territory, team collaborators and product offerings.

- Use online chat, fax, page, voice-mail, video or email to handle workflow of agents and stakeholders.

- Use remote control to see the equipment interface of agents and stakeholders.

- View context-sensitive CTI scripts, popups, FAQs, product update notices, and knowledge bases.

- Understand related contracts, warranties or pertinent service level agreements.

- Generate predictive recommendations. 
- Use instant chat or audio 'whispering 'to coach and train agents.

- Measure and reward agent activity and productivity objectively.

- Obtain lower costs via VOIP versus more costly carrier arrangements.

- Manage home-based agents or off-shore agents with greater scalability and flexibility during seasonal or other peaks and valleys.

\section{ITIL ORIGINS}

Based in the UK, ITIL promotes uniform best practices in methodology frameworks and terminologies. Aspects of the ITIL service management offer techniques to:

- decrease errors and research redundancies;

- aid planning, execution and analysis of IT-related initiatives;

- improve asset availability, reliability, security and continuity;

- define services to support organization per user needs at lowest costs;

- communicate roles and responsibilities;

- analyze key performance indicators;

- apply lessons learned to future operations.

\section{ITIL PRINCIPLES AT CONTACT CENTERS}

IT asset management tools help companies with audit compliance and also reduce the cost of service-desk operations. Technicians gain access to better diagnostic and resolution information. Change and configuration management is substantially enhanced, thus reducing escalations and compressing call times and improving results of problem resolution, release management and change authorizations. Known problems are expeditiously resolved. Root cause analysis and knowledge management repositories are leveraged which helps avoid redundant investigations.

ITIL leverages a configuration database repository (CMDB) to manage a single source of truth for a library of infrastructure details (eg equipment characteristics, contracts and related configuration items). Therefore, the risks of outages, errors or intrusions are better managed. The time required to restore and maintain IT-enabled availability and functionality is reduced.

The ITIL techniques also assist software distribution, version updates and patch distribution.

In many cases, an inventory agent will reside on the client device to collect inventory information about assets that continuously or periodically connect to the network. While installing a lightweight agent is internally straightforward within an organization that owns internal assets, hesitancy may exist on the part of partners, suppliers or customers to permit installing the agent on a device. Hence, a universally trustworthy and non-intrusive agent will be desired. Note that even in the absence of an installed agent on customer devices, a contact center will still benefit from adopting several ITIL principles (eg configuration management database, configuration items, incident handling, definitive software library, etc). Furthermore, the maturity of an organization will influence the readiness and pace for adopting configuration and change management best practices.

When customers are not particularly loyal, or a device is at risk for commoditization, service is an important differentiator and can help diminish need 

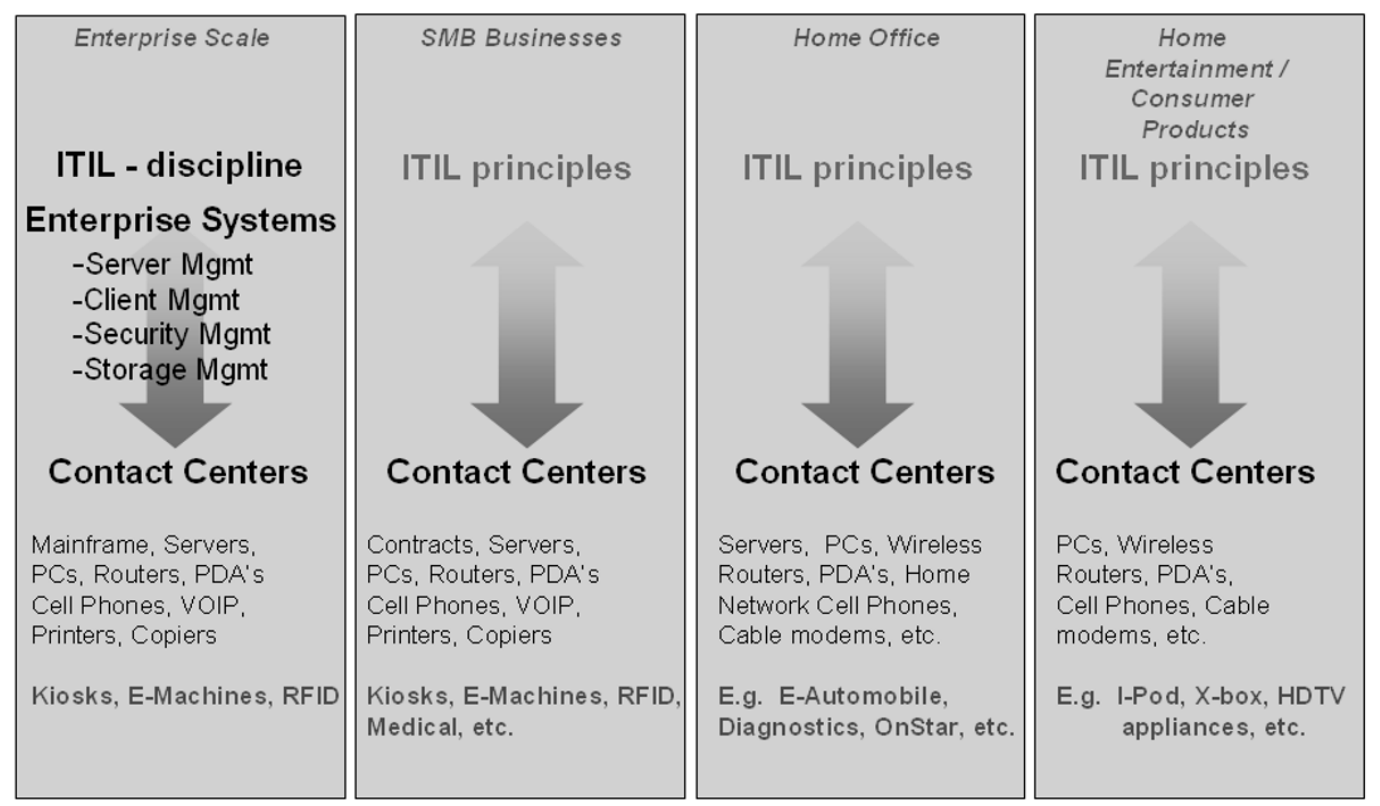

Figure 2: ITIL discipline and change/configuration management

to compete on a basis of price. When MasterCard or Sears provide state-ofthe-art call support, customers' standards and expectations are carried over to firms that may possess fewer resources, or less contact center expertise. Wellknown examples of firms where contact centers have become mission critical include Amazon.com, Federal Express, online retailing, etc.

\section{Summary of ITIL benefits}

- Service quality and reliability:

- customer relationship and customer satisfaction management;

- division of labor;

- efficiency of labor;

- clear definition of responsibilities;

- assignment of role and scope-based authorities;

- process vocabulary: better communication $=$ better coordination $=$ better control.

- Gatekeeper erected for managing changes (release management and change advisory board).

- Deliberate incident management and problem resolution:

- enables roll-back to prior steady state;

- ripple effects and mutual dependencies of networked operations;

- reduce outages, manage mean time to repair (MTTR) and mean time between failures (MTBF);

- continuity management and disaster recovery;

— capacity planning.

- Cost savings:

- incident routing, categorization and handling efficiencies;

- catalogued known problem resolutions;

- avoid redundant research efforts and shared global knowledge bases

- Cost avoidance:

- software contract management; 
- warranty and maintenance contract management (eg expirations, renewals, terminations).

Defining key metrics can be critical to organizations that monitor and maintain service levels. For example, MTTR is used to monitor and trace downtime for interruption incidents. A deliberate approach to maximizing MTBF can help improve uptime and availability (vital to Service Level Agreement Management). Hence, by building and applying service-aware information technology, customer support and repair processes are simplified and automated. Maximizing mean time between incidents (MTBI), for example, leads to improved reliability and uninterrupted revenues. Not missing service thresholds can lower the need to give credit adjustments (rebate penalty) to service contractors.

\section{CONCLUSION}

The comprehensiveness of ITIL principles enables better process discipline, automation and coordination. Labor productivity gains, cost reductions and cost avoidance are far-reaching. Internal facing and external facing contact centers as well as IT-enabled products/services are areas where ITIL renders service level and profit improvements. Applying valuable ITIL principles should not be limited to corporate infrastructure concerns. Outbound and inbound marketing efforts as well as customer operations will benefit. Tuning processes with ITIL helps manage product configurations as well as contact center agents' effectivness. ITIL can help manage $\mathrm{B} 2 \mathrm{~B}$ and $\mathrm{B} 2 \mathrm{C}$ user needs that rely on complex hardware and software configurations. ITIL is not just for infrastrcture. ITIL represents a powerful opportunity to help the business itself. Increasingly, it matters less whether agents are based in Bangalore, Beijing or Boston. The use of ITIL principles in customer-facing contact centers that handle consumer devices can greatly enhance service delivery and service support of e-business and IT enabled products and services.

(C) Cameron A. Fisher

\section{APPENDIX A: GLOSSARY OF TERMS}

Agent: Person employed by a contact center to represent company and perform pre-sales, sales or post-sales customer service. May interact with supplier, partner or management organizations.

Agent: Software that scans and collects inventory and configuration information for IT-enabled PCs, Servers, PDAs and other devices connected to a network.

Asset: Physical or digital property exhibiting value and a productive economic life, including hardware, software, peripherals, etc.

$B S A$ : Business Software Alliance (see www.bsa.org).

Client: End-user personal computer or computing device. Client systems management solutions are often used to manage clients. Server or network systems management solutions are also used by progressive organizations.

$C M D B$ : Configuration management database is a repository of detailed asset data.

Collection: Groupings of computers with a defined common attribute(s). For example, computers "having less than 128K RAM.” 
CTI: Computer telephony integration. Sharing of data and information from computer to enhance telephone operator efficiency. For example, callerID launching display of purchase history and relevant scripting to a CTI user.

ICT: Information and communication technology.

ITAM: Information technology asset management.

ITIL: Information technology infrastructure library.

ITSM: Information technology service management.
Policy: Policies allow actions or triggers to occur when a set of conditions are met.

Predictive analytics: Projects outcomes based on observed patterns of prior occurrences.

Software portal: Allows for software distribution (eg patch, service pack, upgrade) and may permit user selfservice application provisioning or knowledge management.

Wake on LAN: Remote ability to power on, and boot up a client device connected to network.

\section{APPENDIX B: HIGHLIGHTS AND COMPONENTS OF ITIL}

\begin{tabular}{|c|c|c|c|}
\hline Alerts & Requests & Changes & Reports \\
\hline
\end{tabular}

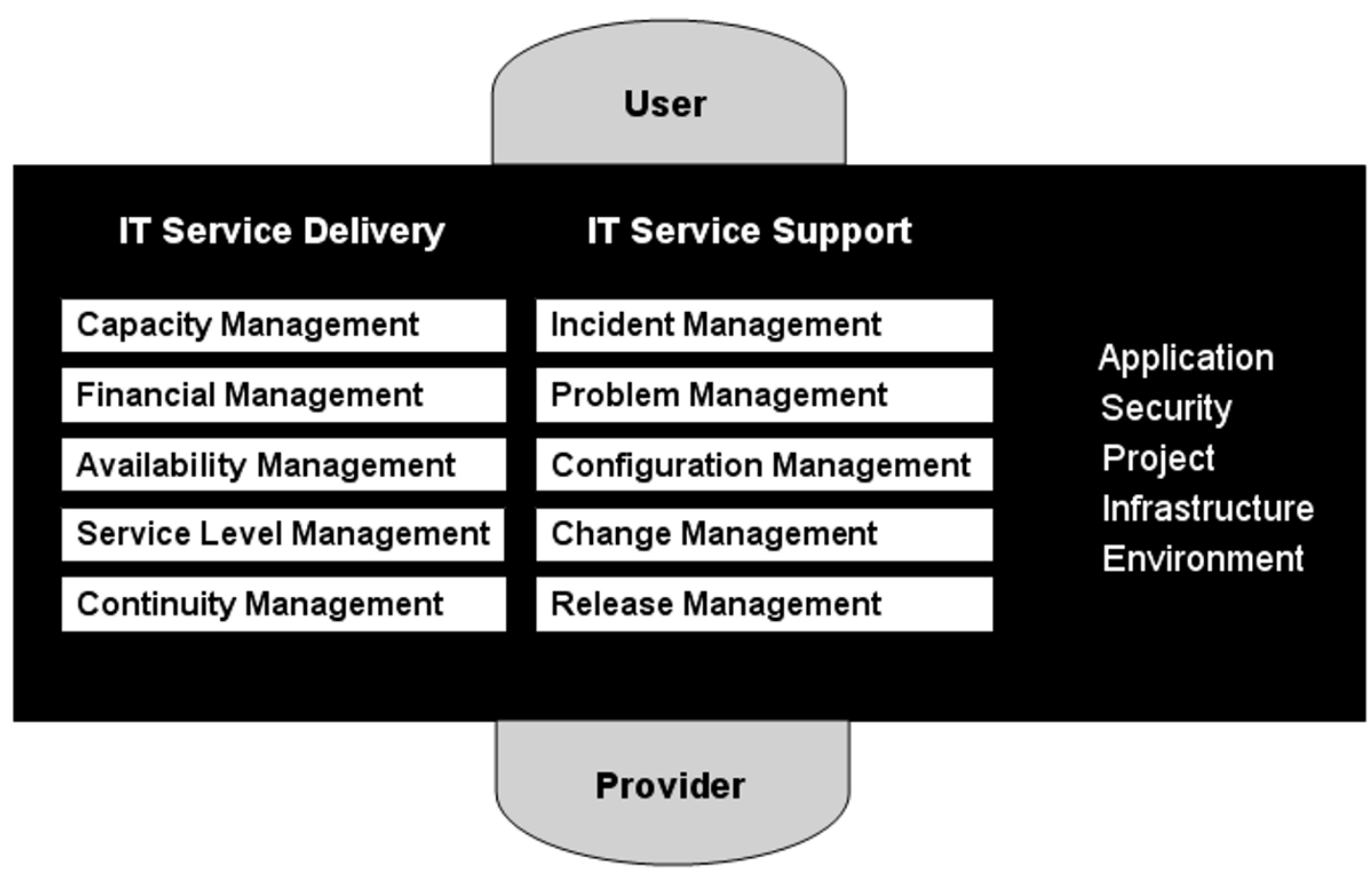

\begin{tabular}{|c|}
\hline Operating Level Agreements \\
\hline Configuration Management \& Detection \\
\hline Infrastructure Management \\
\hline
\end{tabular}




\section{APPENDIX C: THE ITIL FRAMEWORK AND PROCESS FLOW}

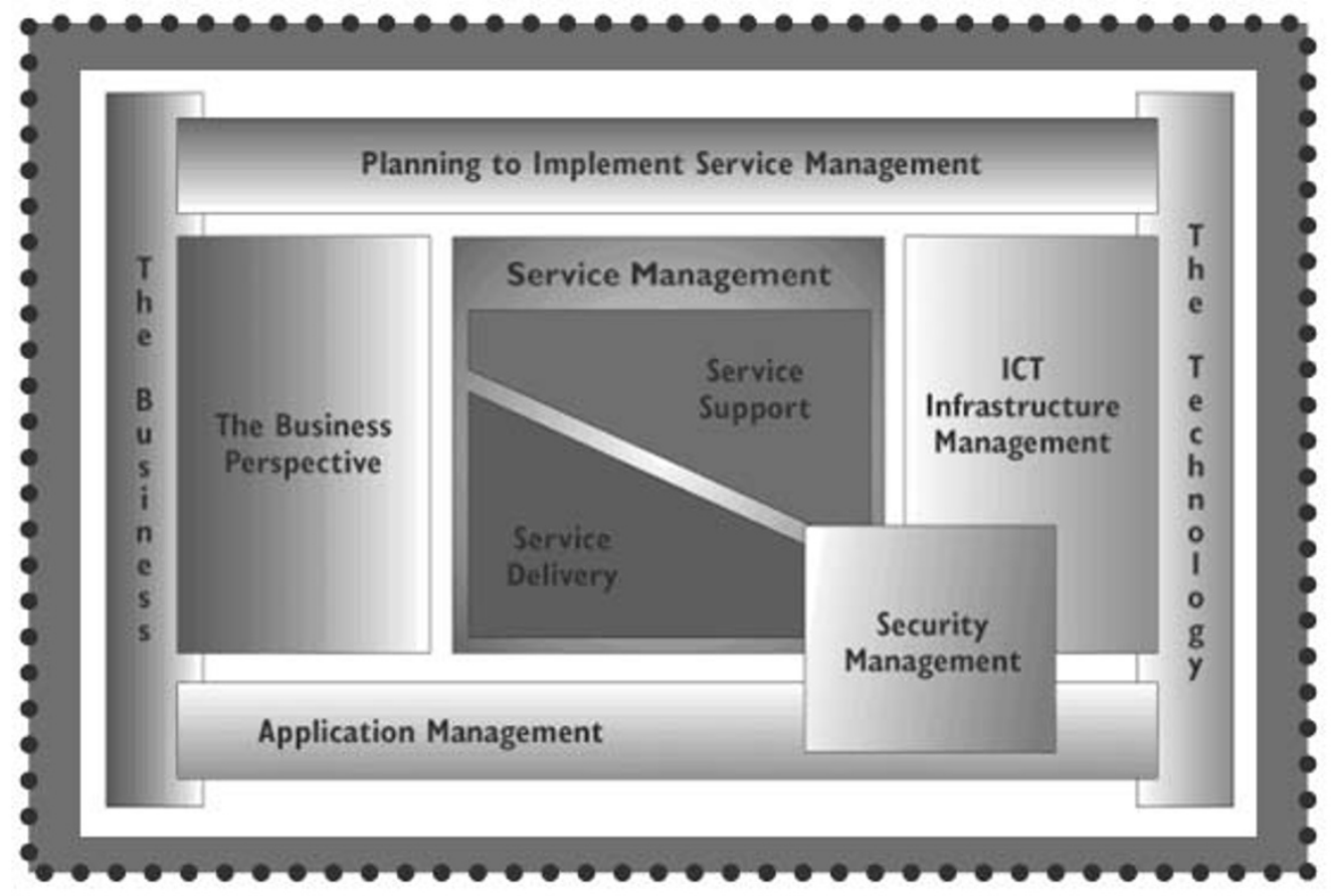

(C) ITIL

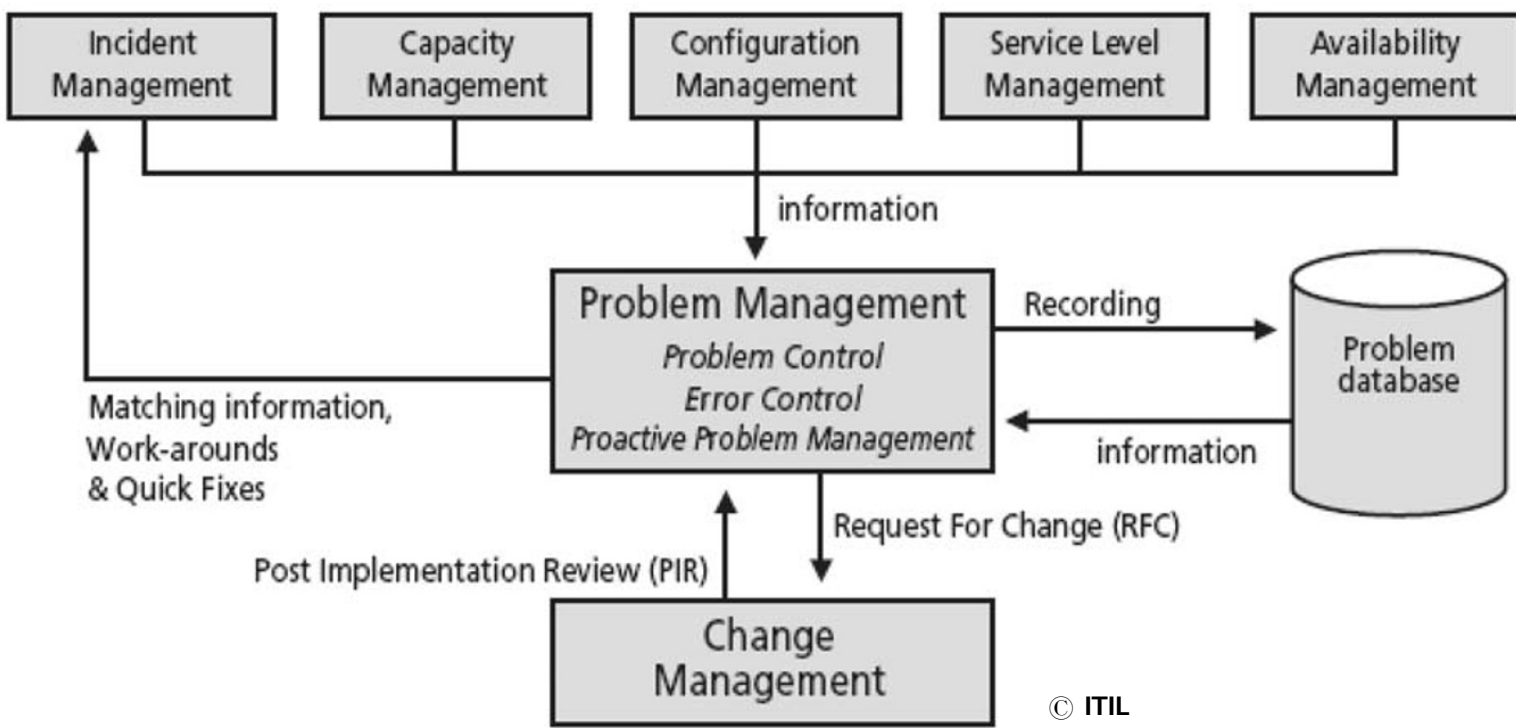




\section{APPENDIX D: HIGHLIGHTS OF ITIL TERMINOLOGY}

The following list includes key concepts that are specific to applying ITIL principles:

Configuration items (CIs): are managed systematically. CIs can vary widely in complexity, size and type. CIs can include an entire system (including all hardware, software and documentation) to a single module, training $\mathrm{CD}$, or peripheral hardware component.

Configuration management database (CMDB): a master database which contains all relevant details of each CI and details of the important relationships between CIs.

Definitive hardware store: helps control authoritative hardware types and related configuration items.

Definitive software library: helps manage version control, service packs, security fixes, etc.

Forward schedule of change: contains details of all changes approved for implementation and their proposed implementation dates. Financial, technical and business implications are examined prior to approval by the change advisory board (CAB). Once agreed externally and internally, the IT service desk communicates to affected users planned downtime arising from scheduled changes using the most effective communication available such as email, e-banners, etc.

Impact analysis: can assess urgency, severity, durations and scope of planned or unplanned events in a systematic manner to better anticipate and react to outages or maintenance.

Incidents: are first sorted, categorized and prioritized prior to attempting to achieve a resolution. A known resolution may already be available. The systematic approach is more effective than individualized or siloed approaches. Root cause analysis strives to resolve incidents to ensure any recurrence is avoided, and to understand any potential interdependencies or roll-back requirements from planned changes.

International standards: ITIL promotes interoperability of vocabulary, skills, methods, configurations and other standards across stakeholders, organizations and industries.

Problem analysis: realizes that an unknown underlying cause of one or more incidents needs to be assessed efficiently for related considerations, known resolutions or related ripple effects. Leveraging the CMDB as a single source of truth leads to important efficiencies.

Release management: enables controlled and gated adjustments to prior steady state. Ability to revert to prior steady state is made more manageable.

Ripple effects are controlled in conjunction with systematic handling of incoming incidents, known or unknown problems and classification and matching of known resolutions to minimize redundant efforts.

Service level agreement (SLA): negotiated understanding between support provider and customer. Describes the services in non-technical terms to enable understanding and periodic adjustment by business unit manager, budget holder or customer. May include menu or catalog of response plans for end-users (eg $7 \times 24$ versus $5 \times 8$ coverage) and alert notification practices. Also related to SLAs are operational level agreements and underpinning contracts. 